\title{
An evaluation of the usefulness of extracorporeal liver support techniques in patients with severe liver dysfunction
}

\author{
Mariusz Piechota ${ }^{1}$, Anna Piechota ${ }^{2}$, Małgorzata Misztal ${ }^{3}$, Szymon Bernas ${ }^{1}$, \\ Iwona Pietraszek-Grzywaczewska ${ }^{1}$
}

\begin{abstract}
${ }^{1}$ Department of Anaesthesiology and Intensive Therapy - Centre for Artificial Extracorporeal Kidney and Liver Support, Dr Wł. Biegański Regional Specialist Hospital, Lodz, Poland

${ }^{2}$ Department of Insurance, Faculty of Economics and Sociology, University of Lodz, Lodz, Poland

${ }^{3}$ Faculty of Economics and Sociology, Chair of Statistical Methods, University of Lodz, Lodz, Poland
\end{abstract}

Submitted: 31 October 2016

Accepted: 2 January 2017

Arch Med Sci 2019; 15, 1: 99-112

DOI: https://doi.org/10.5114/aoms.2017.67998

Copyright $\odot 2017$ Termedia \& Banach

\section{Abstract}

Introduction: The mortality rate in patients with severe liver dysfunction with no option of transplantation is unacceptably high. The main aim of this study was to evaluate the usefulness of applying extracorporeal liver support (ECLS) techniques in this group of patients.

Material and methods: Data from hospital admissions of 101 patients with severe liver dysfunction who were admitted to the department of Anaesthesiology and intensive therapy between 2006 and 2015 were retrospectively analysed. The study group was divided into two subgroups. Standard Medical therapy (SMT) was a subgroup of patients receiving standard Medical therapy, and SMT + ECLS was a subgroup containing patients receiving standard medical therapy complemented by at least one extracorporeal liver support procedure. Results: Significantly lower intensive care unit (ICU) mortality and 30-day mortality rates were found in the SMT + ECLS subgroup $(p=0.0138$ and $p=0.0238$ respectively). No difference in 3 -month mortality was identified between the two groups. In a multivariate model, independent risk factors for ICU mortality proved to be the SOFA score and prothrombin time. The highest discriminatory power for ICU mortality was demonstrated for the SOFA score, followed by APACHE II, SAPS II, MELD UNOS and GCS scores. For 30-day mortality, however, the best discriminatory power was shown for the SAPS II score, followed by SOFA, APACHE II, MELD UNOS and GCS scores. Conclusions: Further studies are needed to assess the contribution of non-biological extracorporeal liver support procedures to a decrease in mortality rates in the population of patients with severe liver dysfunction.

Key words: fractionated plasma separation and adsorption, single pass albumin dialysis, sequential organ failure assessment.

\section{Introduction}

Patients with severe liver dysfunction (MELD UNOS Modification score of 18 or higher) represent an important clinical problem. If standard medical therapy (SMT) is ineffective and the option of liver transplant is not

\author{
Corresponding author: \\ Prof. Mariusz Piechota MD, PhD \\ Department of \\ Anaesthesiology and \\ Intensive Therapy \\ Centre for Artificial \\ Extracorporeal Kidney \\ and Liver Support \\ Dr Wt. Biegański Regional \\ Specialist Hospital \\ $1 / 5$ Kniaziewicza St \\ 91-347 Lodz, Poland \\ Phone: +4842 2516294 \\ E-mail: mariuszpiechota@ \\ poczta.onet.pl
}


available (due to ineligibility for various causes), the mortality rate in this group of patients is unacceptably high [1-3]. In this context, some hope can be placed in non-biological extracorporeal liver support techniques in this group of patients. Extracorporeal liver support (ECLS) can be provided using the fractionated plasma separation and adsorption (FPSA) technique with the Prometheus system, the Molecular Adsorbent Recirculating System (MARS) or single pass albumin dialysis (SPAD) [4-12].

The use of non-biological extracorporeal liver support techniques in patients with severe liver dysfunction is not, strictly speaking, a therapeutic method. However, it is an approach which helps to gain time necessary for the regeneration of the damaged organ, whenever potentially achievable.

There are only limited data available on the benefits of using extracorporeal liver support techniques in patients with severe liver dysfunction [12-14]. One of the main reasons for the scarcity of data seems to be the limited number of studied patients. Another notable fact is that there are no standards applicable to the use of these techniques [7, 11-21].

The main aim of this study was to evaluate the usefulness of applying extracorporeal liver support techniques in this group of patients. The secondary aims were to identify the independent risk factors and to assess the predictive values of the following scoring systems: Glasgow Coma Scale (GCS), Sequential Organ Failure Assessment (SOFA), Acute Physiology and Chronic Health Evaluation II (APACHE II), Simplified Acute Physiology Score II (SAPS II) and Model of End-stage Liver Disease (MELD) United Network for Organ Sharing (UNOS) Modification in patients with severe liver dysfunction.

\section{Material and methods}

\section{Study population and data collection}

We retrospectively analysed the data of 101 patients with severe liver dysfunction (70 men and 31 women) aged between 24 and 83 years who were admitted to the Department of Anaesthesiology and Intensive Therapy (A\&IT) at the Dr Wt. Biegański Regional Specialist Hospital in Lodz between 2006 and 2015. Severe liver dysfunction was defined as a MELD UNOS Modification score of 18 or higher. Where a MELD UNOS score was not included in the medical files, it was determined using an online calculator based on relevant data found in the medical documentation.

The patients were divided into two groups depending on the therapeutic management they received. The SMT subgroup comprised patients who were given standard medical therapy (patients treated in the period 2006-2012). The SMT + ECLS subgroup included patients who received standard medical therapy complemented by at least one extracorporeal liver support procedure (patients treated in the period 2013-2015). Extracorporeal liver support techniques used included FPSA administered with a PROMETHEUS unit or SPAD using MULTIFILTRATE devices.

The medical records of the patients were examined, focusing in particular on the patients' age; sex; reason for A\&IT Department admission; primary and concomitant diseases; GCS and MELD UNOS Modification and DF scores upon admission to the A\&IT Department; SOFA, APACHE II and SAPS II scores on the first day of hospitalization in the A\&IT Department; the duration of stay in the A\&IT Department (in days); and the total duration of hospitalization. The following parameters were also considered: total bilirubin levels, direct bilirubin levels, bile acid levels, ammonia levels, creatinine levels, prothrombin time, prothrombin ratio (INR) and albumin levels prior to the first liver dialysis, as well as the direct bilirubin levels, total bilirubin levels, bile acid levels, ammonia levels and INR after performing the final albumin dialysis.

\section{Extracorporeal liver support techniques administered}

\section{FPSA treatment}

The FPSA eliminates water-soluble and protein-bound toxins and metabolic breakdown products. In the FPSA circuit, venous blood passes through a separator with a pore size of $250 \mathrm{kDa}$ (AlbuFlow, Fresenius AG, Bad Homburg, Germany). The separated plasma then passes through a neutral resin absorbent column (Prometh01, Fresenius AG, Bad Homburg, Germany) and an anion exchange resin absorber (Prometh02, Fresenius AG, Bad Homburg, Germany) to remove albumin-bound toxins. The plasma phase is then returned to the filter and dialyzed as whole blood in a high flow dialyzer (F60S, Fresenius) to remove water-soluble toxins [22]. After a preliminary phase involving a gradual increase in the blood and plasma flow rates in the secondary circuit, which required approximately $12 \mathrm{~min}$, the treatment was performed with a blood flow of $180 \mathrm{ml}$ min and a plasma flow of 270-360 $\mathrm{ml} / \mathrm{min}$. In 1 case, the plasma flow rate in the secondary circuit was increased to $450 \mathrm{ml} / \mathrm{min}$ during the final hours of the procedure to increase the efficacy of FPSA. Sodium citrate was used as an anticoagulant. The typical duration of an individual procedure was between 6 and 10 h. Some individual procedures were completed in less than $6 \mathrm{~h}$ be- 
cause of technical problems (which were likely caused by coagulation on the albumin filter).

\section{Single pass albumin dialysis}

Liver dialysis was performed using the SPAD technique with the aid of standard equipment for continuous venovenous haemodialysis. The patients' blood was dialysed against a standard dialysis solution containing $2 \%$ or $4 \%$ albumin, through a high-flux hollow-fibre haemodiafilter. The treatment was performed with a blood flow rate of $100-200 \mathrm{ml} / \mathrm{min}$ and a dialysate flow rate of $1,000 \mathrm{ml} / \mathrm{min}$. The dialysate, which was enriched with albumin following a single contact with the patient's blood in the haemofilter, was drained into the waste bag and disposed of. The procedure used $10,000 \mathrm{ml}$ of dialysate containing $2 \%$ albumin or $5,000 \mathrm{ml}$ of dialysate containing $4 \%$ albumin. Sodium citrate was used as an anticoagulant. Depending on the albumin concentration in the dialysate, the duration of a single procedure was between 5 and $10 \mathrm{~h}$.

\section{Statistical analysis}

All the calculations were performed using Statistica (StatSoft, Inc. (2009), Statistica (data analysis software system), version 10, Tulsa, USA) and SPSS (IBM SPSS Statistics, version 21, USA) software.

Quantitative variables were expressed as mean \pm SD (standard deviation). Minimum, maximum, median and interquartile range (IQR: Q25-Q75) were also presented. For categorical variables the number of observations $(N)$ and corresponding percentages (\%) were calculated.

Normality was tested using the Shapiro-Wilk test for normality.

Differences between two independent samples for continuous data were analysed with the Mann-Whitney $U$ test (if the distributions of variables were different from normal) or Student's $t$-test (for normal distributions). For categorical variables, the statistical analysis was based either on the $\chi^{2}$ test or the $\chi^{2}$ test with Yates' adjustment.

Wilcoxon signed rank test or Student's $t$-test for two dependent samples was applied to compare patients' characteristics on admission and discharge from the hospital. Two-way ANOVA with repeated measures was used if more factors were taken into consideration.

For quantitative variables significantly associated with mortality, ROC curves were drawn and decision thresholds were determined (based on Youden's index). Sensitivity, specificity, positive predictive value (PPV) and negative predictive value (NPV) were calculated. The area under the ROC curve (AUC) with SE (standard error), 95\% Cl and corresponding $p$-value were also presented.
A multivariate forward stepwise logistic regression model was used to identify the set of independent risk factors for mortality.

The results were considered significant for $p<0.05$.

\section{Ethics statement}

The study was approved by the Bioethics Committee of the Medical University of Lodz (RNN/297/15/KE). Because of the retrospective nature of the study and according to the Polish law, no informed consent was required.

\section{Results}

Among 101 patients included in the study, 49 patients were diagnosed with hepatic encephalopathy, 24 with hepatorenal syndrome, 60 with cirrhosis with ascites and 59 with alcoholic liver disease. The group included 70 (69.31\%) men. A total of 67 patients died during their intensive care unit (ICU) stay (66.34\%).

The SMT subgroup included 53 patients (17 women and 36 men), and the SMT + ECLS subgroup included 48 patients (14 women and 34 men). Descriptive statistics for the study group and the SMT and SMT + ECLS subgroups are listed in Table I.

Significantly lower ICU mortality and 30-day mortality rates were found in the SMT + ECLS subgroup. No difference in 3-month mortality was identified between the two groups (Table II).

Since significant differences in ICU mortality and 30-day mortality were found between the study groups, the inclusion in a particular group was regarded as a death risk factor, and multidimensional models were used to determine whether it represented an independent risk factor.

\section{Risk of ICU mortality}

Compared to ICU survivors, the patients who died in the ICU were older, had lower GCS scores and lower serum albumin levels, higher SOFA, APACHE II, SAPS II and MELD UNOS scores as well as higher INR, prothrombin time and creatinine levels. Furthermore, the group of patients who died in the ICU included more cases of hepatic encephalopathy, cirrhosis with ascites and hepatorenal syndrome. Also, they more commonly received continuous renal replacement therapy (CRRT) than ECLS treatment. For the remaining variables, no statistically significant differences were found (Table III).

Consequently, the following parameters were considered as potential risk factors for ICU mortality: patient age (years), GCS (score), SOFA (score), APACHE II (score), SAPS II (score), MELD UNOS Modification (score), INR on ICU admission, prothrombin time (s) on ICU admission, creatinine (mg/dl) on ICU admission, and serum albumin 
Table I. Characteristics of the study group and subgroups: a subgroup of patients receiving standard medical therapy, and a subgroup containing patients receiving standard medical therapy complemented by at least one extracorporeal liver support procedure

\begin{tabular}{|c|c|c|c|c|c|}
\hline Variable & & Total $(N=101)$ & $\begin{array}{l}\text { SMT group } \\
(N=53)\end{array}$ & $\begin{array}{c}\text { SMT + ECLS group } \\
(N=48)\end{array}$ & $\begin{array}{c}p \text {-value } \\
\text { (SMT group } \\
\text { vs. SMT + } \\
\text { ECLS group) }\end{array}$ \\
\hline Age [years] & $\begin{array}{c}\text { Mean } \pm \text { SD } \\
\text { Me (IQR) } \\
\text { Min.-max. }\end{array}$ & $\begin{array}{c}47.89 \pm 13.41 \\
49(38-57) \\
24-83\end{array}$ & $\begin{array}{c}51.26 \pm 12.40 \\
51(44-59) \\
27-83\end{array}$ & $\begin{array}{c}44.17 \pm 13.62 \\
43.5(34.5-55.5) \\
24-73\end{array}$ & 0.0073 \\
\hline \multicolumn{6}{|c|}{ Etiology of severe liver dysfunction: } \\
\hline $\begin{array}{l}\text { Alcoholic } \\
\text { Viral } \\
\text { Drugs } \\
\text { Other } \\
\text { Mixed } \\
\text { Unknown }\end{array}$ & $\begin{array}{l}N(\%) \\
N(\%) \\
N(\%) \\
N(\%) \\
N(\%) \\
N(\%)\end{array}$ & $\begin{aligned} 56 & (55.45) \\
11 & (10.89) \\
4 & (3.96) \\
11 & (10.89) \\
4 & (3.96) \\
15 & (14.85)\end{aligned}$ & $\begin{array}{c}27(50.94) \\
9(16.98) \\
3(5.66) \\
4(7.55) \\
2(3.77) \\
8(15.09)\end{array}$ & $\begin{array}{c}29(60.42) \\
2(4.17) \\
1(2.08) \\
7(14.58) \\
2(4.17) \\
7(14.58)\end{array}$ & \\
\hline $\begin{array}{l}\text { Hepatic } \\
\text { encephalopathy }\end{array}$ & $N(\%)$ & $49(49.00)$ & $27(50.94)$ & $22(46.81)$ & 0.6797 \\
\hline $\begin{array}{l}\text { Cirrhosis with } \\
\text { ascites }\end{array}$ & $N(\%)$ & $60(59.41)$ & $34(64.15)$ & $26(54.17)$ & 0.3075 \\
\hline $\begin{array}{l}\text { Hepatorenal } \\
\text { syndrome }\end{array}$ & $N(\%)$ & $24(23.76)$ & $6(11.32)$ & $18(37.50)$ & 0.0017 \\
\hline $\begin{array}{l}\text { Alcoholic liver } \\
\text { disease }\end{array}$ & $N(\%)$ & $59(60.20)$ & $29(58.00)$ & $30(62.50)$ & 0.6491 \\
\hline GCS score & $\begin{array}{l}\text { Mean } \pm \text { SD } \\
\text { Me (IQR) } \\
\text { Min.-max. }\end{array}$ & $\begin{array}{c}10.48 \pm 4.73 \\
13(5-15) \\
3-15\end{array}$ & $\begin{array}{c}7.72 \pm 4.32 \\
7(4-12) \\
3-15\end{array}$ & $\begin{array}{c}13.52 \pm 3.00 \\
15(14-15) \\
3-15\end{array}$ & $<0.0001$ \\
\hline SOFA score & $\begin{array}{c}\text { Mean } \pm \text { SD } \\
\text { Me (IQR) } \\
\text { Min.-max. }\end{array}$ & $\begin{array}{c}13.68 \pm 4.42 \\
14(11-17) \\
5-22\end{array}$ & $\begin{array}{c}15.49 \pm 3.20 \\
15(13-17) \\
10-22\end{array}$ & $\begin{array}{c}11.59 \pm 4.73 \\
12.5(7-14) \\
5-21\end{array}$ & $<0.0001$ \\
\hline APACHE II score & $\begin{array}{c}\text { Mean } \pm \text { SD } \\
\text { Me }(I Q R) \\
\text { Min.-max. }\end{array}$ & $\begin{array}{c}25.39 \pm 9.99 \\
25(18-32) \\
4-55 \\
\end{array}$ & $\begin{array}{c}29.94 \pm 8.15 \\
29(24-35) \\
15-55 \\
\end{array}$ & $\begin{array}{c}20.15 \pm 9.39 \\
18.5(14-25) \\
4-43 \\
\end{array}$ & $<0.0001$ \\
\hline SAPS II score & $\begin{array}{l}\text { Mean } \pm \text { SD } \\
\text { Me (IQR) } \\
\text { Min.-max. }\end{array}$ & $\begin{array}{c}54.84 \pm 22.16 \\
54(37-71) \\
15-103\end{array}$ & $\begin{array}{c}66.21 \pm 17.55 \\
66(56-78) \\
28-103\end{array}$ & $\begin{array}{c}41.74 \pm 19.64 \\
40(27-52) \\
15-95\end{array}$ & $<0.0001$ \\
\hline $\begin{array}{l}\text { MELD UNOS } \\
\text { Modification } \\
\text { score }\end{array}$ & $\begin{array}{c}\text { Mean } \pm \text { SD } \\
\text { Me (IQR) } \\
\text { Min.-max. }\end{array}$ & $\begin{array}{c}31.63 \pm 8.15 \\
31(25-39) \\
18-48\end{array}$ & $\begin{array}{c}30.58 \pm 8.13 \\
29(23-37) \\
18-48\end{array}$ & $\begin{array}{c}32.79 \pm 8.09 \\
32.5(26-39) \\
18-48\end{array}$ & 0.1681 \\
\hline $\begin{array}{l}\text { Length of } \\
\text { hospital stay } \\
\text { [days] }\end{array}$ & $\begin{array}{c}\text { Mean } \pm \text { SD } \\
\text { Me (IQR) } \\
\text { Min.-max. }\end{array}$ & $\begin{array}{c}21.25 \pm 16.41 \\
18(7-29) \\
1-63\end{array}$ & $\begin{array}{c}19.85 \pm 16.76 \\
16(7-29) \\
1-63\end{array}$ & $\begin{array}{c}22.79 \pm 16.04 \\
20(9.5-29.5) \\
3-60\end{array}$ & 0.2645 \\
\hline $\begin{array}{l}\text { Length of ICU } \\
\text { stay [days] }\end{array}$ & $\begin{array}{c}\text { Mean } \pm \text { SD } \\
\text { Me (IQR) } \\
\text { Min.-max. }\end{array}$ & $\begin{array}{c}10.80 \pm 10.63 \\
7(4-13) \\
1-51\end{array}$ & $\begin{array}{c}9.66 \pm 10.32 \\
7(2-12) \\
1-48 \\
\end{array}$ & $\begin{array}{c}12.06 \pm 10.92 \\
8.5(5-13.5) \\
3-51\end{array}$ & 0.0643 \\
\hline $\begin{array}{l}\text { Total bilirubin } \\
{[\mathrm{mg} / \mathrm{dl}]}\end{array}$ & $\begin{array}{l}\text { Mean } \pm \text { SD } \\
\text { Me }(I Q R) \\
\text { Min.-max. }\end{array}$ & $\begin{array}{c}18.91 \pm 12.60 \\
18.77 \\
(7.07-28.48) \\
0.66-45.54\end{array}$ & $\begin{array}{c}10.55 \pm 8.89 \\
7.27 \\
(4.35-15.83) \\
0.66-36.8 \\
\end{array}$ & $\begin{array}{c}28.14 \pm 9.20 \\
28.15 \\
(21.87-35.70) \\
2.71-45.54\end{array}$ & $<0.0001$ \\
\hline $\begin{array}{l}\text { Direct bilirubin } \\
{[\mathrm{mg} / \mathrm{dl}]}\end{array}$ & $\begin{array}{c}\text { Mean } \pm \text { SD } \\
\text { Me }(I Q R) \\
\text { Min.-max. }\end{array}$ & $\begin{array}{c}21.62 \pm 9.47 \\
21.3(14.8-29.14) \\
0.71-43.21 \\
\end{array}$ & $\begin{array}{c}12.32 \pm 7.60 \\
12.83(6.48-14.8) \\
0.71-28.26 \\
\end{array}$ & $\begin{array}{c}23.75 \pm 8.58 \\
22.69(16.63-29.65) \\
2.08-43.21 \\
\end{array}$ & 0.0001 \\
\hline $\begin{array}{l}\text { Bile acids } \\
{[\mu \mathrm{mol} / \mathrm{l}]}\end{array}$ & $\begin{array}{c}\text { Mean } \pm \text { SD } \\
\text { Me }(I Q R) \\
\text { Min.-max. }\end{array}$ & $\begin{array}{c}113.96 \pm 93.28 \\
93.2(61.1-138.7) \\
28.4-549.3\end{array}$ & $\begin{array}{c}67.60 \pm 25.07 \\
70.6(65.7-75.1) \\
28.6-98\end{array}$ & $\begin{array}{c}119.00 \pm 96.68 \\
94.80(61.10-140.60) \\
28.40-549.30\end{array}$ & 0.1408 \\
\hline $\begin{array}{l}\text { Ammonia } \\
[\mu \mathrm{g} / \mathrm{dl}])\end{array}$ & $\begin{array}{l}\text { Mean } \pm \text { SD } \\
\text { Me (IQR) } \\
\text { Min.-max. }\end{array}$ & $\begin{array}{c}149.78 \pm 196.80 \\
96.5(70-148) \\
13-1535\end{array}$ & $\begin{array}{c}194.3 \pm 256.49 \\
112(73-191) \\
24-1535\end{array}$ & $\begin{array}{c}105.34 \pm 92.07 \\
83(62-135) \\
13-620\end{array}$ & 0.5138 \\
\hline
\end{tabular}


Table I. Cont.

\begin{tabular}{|c|c|c|c|c|c|}
\hline Variable & & Total $(N=101)$ & $\begin{array}{l}\text { SMT group } \\
(N=53)\end{array}$ & $\begin{array}{l}\text { SMT + ECLS group } \\
(N=48)\end{array}$ & $\begin{array}{c}p \text {-value } \\
\text { (SMT group } \\
\text { vs. SMT + } \\
\text { ECLS group) }\end{array}$ \\
\hline INR & $\begin{array}{l}\text { Mean } \pm \text { SD } \\
\text { Me (IQR) } \\
\text { Min.-max. }\end{array}$ & $\begin{array}{c}2.42 \pm 0.94 \\
2.27(1.64-3.07) \\
0.86-5.46\end{array}$ & $\begin{array}{c}2.48 \pm 0.94 \\
2.31(1.66-3.17) \\
1.23-4.73\end{array}$ & $\begin{array}{c}2.35 \pm 0.95 \\
2.16(1.62-2.86) \\
0.86-5.46\end{array}$ & 0.0314 \\
\hline $\begin{array}{l}\text { Prothrombin } \\
\text { time }[\mathrm{s}]\end{array}$ & $\begin{array}{l}\text { Mean } \pm \text { SD } \\
\text { Me (IQR) } \\
\text { Min.-max. }\end{array}$ & $\begin{array}{c}30.16 \pm 19.74 \\
26.6(18.4-36.9) \\
9.9-189.7 \\
\end{array}$ & $\begin{array}{c}32.82 \pm 25.00 \\
27.9(18.4-39.88) \\
14.4-189.7 \\
\end{array}$ & $\begin{array}{c}27.23 \pm 11.00 \\
25.60(18.65-32.45) \\
9.90-63.90\end{array}$ & 0.2826 \\
\hline $\begin{array}{l}\text { Creatinine } \\
{[\mathrm{mg} / \mathrm{dl}]}\end{array}$ & $\begin{array}{l}\text { Mean } \pm \text { SD } \\
\text { Me (IQR) } \\
\text { Min.-max. }\end{array}$ & $\begin{array}{c}2.58 \pm 2.27 \\
1.85(0.9-3.57) \\
0.1-13.52\end{array}$ & $\begin{array}{c}2.88 \pm 2.45 \\
2.39(1.1-4.11) \\
0.33-13.52\end{array}$ & $\begin{array}{c}2.25 \pm 2.02 \\
1.59(0.77-3.07) \\
0.10-7.76\end{array}$ & 0.0316 \\
\hline Albumin [g/dl] & $\begin{array}{l}\text { Mean } \pm \text { SD } \\
\text { Me (IQR) } \\
\text { Min.-max. }\end{array}$ & $\begin{array}{c}2.77 \pm 0.68 \\
2.88(2.21-3.21) \\
0.9-4.47\end{array}$ & $\begin{array}{c}2.60 \pm 0.73 \\
2.545(2.11-3.1) \\
0.9-4.16\end{array}$ & $\begin{array}{c}2.93 \pm 0.61 \\
3.00(2.47-3.21) \\
1.80-4.47\end{array}$ & 0.0793 \\
\hline Liver dialyses & $\begin{array}{l}\text { Mean } \pm \text { SD } \\
\text { Me (IQR) } \\
\text { Min.-max. }\end{array}$ & & - & $\begin{array}{c}4.35 \pm 2.78 \\
3(3-6) \\
1-12\end{array}$ & \\
\hline FPSA & $\begin{array}{l}\text { Mean } \pm \text { SD } \\
\text { Me }(I Q R) \\
\text { Min.-max. }\end{array}$ & & - & $\begin{array}{c}0.35 \pm 0.91 \\
0(0-0) \\
0-5\end{array}$ & \\
\hline SPAD & $\begin{array}{l}\text { Mean } \pm \text { SD } \\
\text { Me (IQR) } \\
\text { Min.-max. }\end{array}$ & & - & $\begin{array}{c}4.00 \pm 2.63 \\
3(2-5.5) \\
0-12\end{array}$ & \\
\hline $\begin{array}{l}\text { CRRT during ICU } \\
\text { stay }\end{array}$ & $N(\%)$ & $42(41.58)$ & 17 (32.08) & $25(52.08)$ & 0.0416 \\
\hline
\end{tabular}

APACHE - Acute Physiology and Chronic Health Evaluation, CRRT - continuous renal replacement therapy, ECLS - extracorporeal liver support, GCS - Glasgow Coma Scale, FPSA - fractionated plasma separation and adsorption, ICU - intensive care unit, INR - prothrombin ratio, IQR - interquartile range, MELD UNOS - Model of End-stage Liver Disease United Network for Organ Sharing, SAPS - Simplified Acute Physiology Score, SD - standard deviation, SMT - standard medical therapy, SOFA - Sequential Organ Failure Assessment, SPAD - single pass albumin dialysis.

Table II. Comparison of mortality between a subgroup of patients receiving standard medical therapy, and a subgroup containing patients receiving standard medical therapy complemented by at least one extracorporeal liver support procedure

\begin{tabular}{|c|c|c|c|c|c|c|c|}
\hline \multirow[t]{2}{*}{ Group } & & \multicolumn{2}{|c|}{ ICU mortality } & \multicolumn{2}{|c|}{ 30-day mortality } & \multicolumn{2}{|c|}{ 3-month mortality } \\
\hline & & $\begin{array}{c}\text { Patients } \\
\text { who survived }\end{array}$ & $\begin{array}{l}\text { Patients } \\
\text { who died }\end{array}$ & $\begin{array}{c}\text { Patients } \\
\text { who survived }\end{array}$ & $\begin{array}{l}\text { Patients } \\
\text { who died }\end{array}$ & $\begin{array}{c}\text { Patients } \\
\text { who survived }\end{array}$ & $\begin{array}{l}\text { Patients } \\
\text { who died }\end{array}$ \\
\hline \multirow[t]{2}{*}{$\mathrm{SMT}$} & $N$ & 12 & 41 & 9 & 44 & 7 & 46 \\
\hline & $\%$ & 22.64 & 77.36 & 16.98 & 83.02 & 13.21 & 86.79 \\
\hline \multirow{2}{*}{$\begin{array}{l}\text { SMT + } \\
\text { ECLS }\end{array}$} & $N$ & 22 & 26 & 17 & 29 & 11 & 34 \\
\hline & $\%$ & 45.83 & 54.17 & 36.96 & 63.04 & 24.44 & 75.56 \\
\hline Total & & 34 & 67 & 26 & 73 & 18 & 80 \\
\hline$P$-value & & \multicolumn{2}{|c|}{0.0138} & \multicolumn{2}{|c|}{0.0238} & \multicolumn{2}{|c|}{0.1523} \\
\hline
\end{tabular}

ECLS - extracorporeal liver support, ICU - intensive care unit, SMT - standard medical therapy.

level on ICU admission (g/dl). For these variables, ROC curves were drawn in order to assess discriminatory power (Table IV).

Based on Youden's index, optimum cut-off points were determined. For them, basic performance metrics which evaluate the resulting diagnostic tests suitable for predicting ICU mortality were calculated (Table V).
The highest values of all four measures (sensitivity, specificity, PPV and NPV) were determined for SOFA $\geq 10.5$, SAPS $\| \geq 39.5$ and APACHE $\| \geq 21$.

Univariate logistic regression identified the following risk factors for ICU mortality in patients with severe liver dysfunction: age $(p<0.009$; $\mathrm{OR}=1.046,95 \% \mathrm{Cl}: 1.011-1.083)$, SOFA $(p<0.001$; $\mathrm{OR}=1.547,95 \% \mathrm{Cl}: 1.299-1.841)$, APACHE ॥ 
Mariusz Piechota, Anna Piechota, Małgorzata Misztal, Szymon Bernas, Iwona Pietraszek-Grzywaczewska

Table III. The ICU mortality. Descriptive characteristics of survivors and deceased patients

\begin{tabular}{|c|c|c|c|c|}
\hline Variable & & Survivors $(N=34)$ & Deceased patients $(N=67)$ & $P$-value \\
\hline Age [years] & $\begin{array}{l}\text { Mean } \pm \text { SD } \\
\text { Me (IQR) } \\
\text { Min.-max. }\end{array}$ & $\begin{array}{c}42.91 \pm 13.63 \\
43(31-51) \\
24-78\end{array}$ & $\begin{array}{c}50.42 \pm 12.66 \\
52(43-59) \\
27-83 \\
\end{array}$ & 0.0095 \\
\hline $\begin{array}{l}\text { Hepatic } \\
\text { encephalopathy }\end{array}$ & $N(\%)$ & $12(35.29)$ & $37(56.06)$ & 0.0491 \\
\hline $\begin{array}{l}\text { Cirrhosis with } \\
\text { ascites }\end{array}$ & $N(\%)$ & $14(41.18)$ & $46(68.66)$ & 0.0079 \\
\hline $\begin{array}{l}\text { Hepatorenal } \\
\text { syndrome }\end{array}$ & $N(\%)$ & $3(8.82)$ & $21(31.34)$ & 0.0235 \\
\hline $\begin{array}{l}\text { Alcoholic liver } \\
\text { disease }\end{array}$ & $N(\%)$ & $20(60.61)$ & $39(60.00)$ & 0.9538 \\
\hline GCS score & $\begin{array}{l}\text { Mean } \pm \text { SD } \\
\text { Me (IQR) } \\
\text { Min.-max. }\end{array}$ & $\begin{array}{c}12.18 \pm 4.11 \\
15(9-15) \\
3-15 \\
\end{array}$ & $\begin{array}{c}9.61 \pm 4.82 \\
11(5-14) \\
3-15 \\
\end{array}$ & 0.0016 \\
\hline SOFA score & $\begin{array}{l}\text { Mean } \pm \text { SD } \\
\text { Me (IQR) } \\
\text { Min.-max. }\end{array}$ & $\begin{array}{c}9.78 \pm 4.12 \\
8(6.5-13) \\
5-17 \\
\end{array}$ & $\begin{array}{c}15.54 \pm 3.19 \\
15(13-18) \\
10-22 \\
\end{array}$ & $<0.0001$ \\
\hline APACHE II score & $\begin{array}{l}\text { Mean } \pm \text { SD } \\
\text { Me (IQR) } \\
\text { Min.-max. }\end{array}$ & $\begin{array}{c}17.72 \pm 7.80 \\
17(13-21) \\
4-36 \\
\end{array}$ & $\begin{array}{c}29.06 \pm 8.81 \\
29(23-36) \\
12-55 \\
\end{array}$ & $<0.0001$ \\
\hline SAPS II score & $\begin{array}{l}\text { Mean } \pm \text { SD } \\
\text { Me (IQR) } \\
\text { Min.-max. }\end{array}$ & $\begin{array}{c}37.66 \pm 18.86 \\
31(25-52) \\
15-81\end{array}$ & $\begin{array}{c}63.04 \pm 18.73 \\
64(48-73) \\
22-103\end{array}$ & $<0.0001$ \\
\hline $\begin{array}{l}\text { MELD UNOS } \\
\text { Modification } \\
\text { score }\end{array}$ & $\begin{array}{l}\text { Mean } \pm \text { SD } \\
\text { Me (IQR) } \\
\text { Min.-max. }\end{array}$ & $\begin{array}{c}27.15 \pm 5.49 \\
26.5(23-31) \\
18-40\end{array}$ & $\begin{array}{c}33.91 \pm 8.36 \\
35(27-41) \\
18-48\end{array}$ & 0.0001 \\
\hline $\begin{array}{l}\text { Length of } \\
\text { hospital stay } \\
\text { [days] }\end{array}$ & $\begin{array}{l}\text { Mean } \pm \text { SD } \\
\text { Me (IQR) } \\
\text { Min.-max. }\end{array}$ & $\begin{array}{c}26.82 \pm 16.81 \\
26(14-38) \\
3-60\end{array}$ & $\begin{array}{c}18.42 \pm 15.57 \\
14(7-28) \\
1-63\end{array}$ & 0.0113 \\
\hline $\begin{array}{l}\text { Length of ICU } \\
\text { stay [days] }\end{array}$ & $\begin{array}{l}\text { Mean } \pm \text { SD } \\
\text { Me (IQR) } \\
\text { Min.-max. }\end{array}$ & $\begin{array}{c}11.32 \pm 11.23 \\
7(5-12) \\
3-48\end{array}$ & $\begin{array}{c}10.54 \pm 10.38 \\
8(3-14) \\
1-51\end{array}$ & 0.6761 \\
\hline $\begin{array}{l}\text { Total bilirubin } \\
{[\mathrm{mg} / \mathrm{dl}]}\end{array}$ & $\begin{array}{l}\text { Mean } \pm \text { SD } \\
\text { Me (IQR) } \\
\text { Min.-max. }\end{array}$ & $\begin{array}{c}21.95 \pm 13.25 \\
22.03(9.47-30.48) \\
0.66-45.54\end{array}$ & $\begin{array}{c}17.37 \pm 12.07 \\
17.51(6.15-25.71) \\
0.69-43.22\end{array}$ & 0.0913 \\
\hline $\begin{array}{l}\text { Direct bilirubin } \\
{[\mathrm{mg} / \mathrm{dl}]}\end{array}$ & $\begin{array}{l}\text { Mean } \pm \text { SD } \\
\text { Me (IQR) } \\
\text { Min.-max. }\end{array}$ & $\begin{array}{c}23.66 \pm 9.58 \\
23.98(15.51-29.44) \\
2.08-43.21 \\
\end{array}$ & $\begin{array}{c}20.11 \pm 9.25 \\
20.96(13.24-28.26) \\
0.71-42.85\end{array}$ & 0.1592 \\
\hline $\begin{array}{l}\text { Bile acids } \\
{[\mu \mathrm{mol} / \mathrm{l}]}\end{array}$ & $\begin{array}{l}\text { Mean } \pm \text { SD } \\
\text { Me (IQR) } \\
\text { Min.-max. }\end{array}$ & $\begin{array}{c}127.80 \pm 106.95 \\
96.2(79.9-133) \\
28.7-549.3\end{array}$ & $\begin{array}{c}100.66 \pm 77.75 \\
76.65(51.8-138.7) \\
28.4-415.8\end{array}$ & 0.1549 \\
\hline Ammonia [ $\mu \mathrm{g} / \mathrm{dl}]$ & $\begin{array}{l}\text { Mean } \pm \text { SD } \\
\text { Me (IQR) } \\
\text { Min.-max. }\end{array}$ & $\begin{array}{c}107.75 \pm 87.99 \\
94(67-123) \\
16-543.6 \\
\end{array}$ & $\begin{array}{c}172.52 \pm 233.25 \\
103.6(72-165) \\
13-1535 \\
\end{array}$ & 0.2743 \\
\hline INR & $\begin{array}{l}\text { Mean } \pm \text { SD } \\
\text { Me (IQR) } \\
\text { Min.-max. }\end{array}$ & $\begin{array}{c}1.91 \pm 0.60 \\
1.88(1.5-2.31) \\
0.86-3.51 \\
\end{array}$ & $\begin{array}{c}2.67 \pm 0.98 \\
2.59(1.8-3.45) \\
1.24-5.46 \\
\end{array}$ & 0.0002 \\
\hline $\begin{array}{l}\text { Prothrombin } \\
\text { time }[\mathrm{s}]\end{array}$ & $\begin{array}{l}\text { Mean } \pm \text { SD } \\
\text { Me (IQR) } \\
\text { Min.-max. }\end{array}$ & $\begin{array}{c}22.24 \pm 6.66 \\
21.85(17.2-27.2) \\
9.9-40.4\end{array}$ & $\begin{array}{c}34.19 \pm 22.80 \\
30.7(20.9-42.9) \\
14.4-189.7\end{array}$ & 0.0001 \\
\hline $\begin{array}{l}\text { Creatinine } \\
{[\mathrm{mg} / \mathrm{dl}]}\end{array}$ & $\begin{array}{c}\text { Mean } \pm \text { SD } \\
\text { Me (IQR) } \\
\text { Min.-max. }\end{array}$ & $\begin{array}{c}1.65 \pm 1.60 \\
1(0.74-1.8) \\
0.39-6.81\end{array}$ & $\begin{array}{c}3.05 \pm 2.42 \\
2.58(1.35-4.13) \\
0.1-13.52\end{array}$ & 0.0002 \\
\hline Albumin $[\mathrm{g} / \mathrm{dl}]$ & $\begin{array}{l}\text { Mean } \pm \text { SD } \\
\text { Me (IQR) } \\
\text { Min.-max. }\end{array}$ & $\begin{array}{c}3.11 \pm 0.60 \\
3.10(2.835-3.515) \\
1.45-4.47\end{array}$ & $\begin{array}{c}2.58 \pm 0.66 \\
2.53(2.09-3.09) \\
0.9-4.16\end{array}$ & 0.0003 \\
\hline
\end{tabular}


Table III. Cont.

\begin{tabular}{|lcccc|}
\hline Variable & & Survivors $(\boldsymbol{N}=34)$ & Deceased patients $(\boldsymbol{N}=67)$ & $P$-value \\
\hline $\begin{array}{l}\text { ECLS during ICU } \\
\text { stay }\end{array}$ & $N(\%)$ & $22(64.71)$ & $26(38.81)$ & 0.0138 \\
\hline $\begin{array}{l}\text { CRRT during ICU } \\
\text { stay }\end{array}$ & $N(\%)$ & $8(23.53)$ & $34(50.75)$ & 0.0074 \\
\hline
\end{tabular}

APACHE - Acute Physiology and Chronic Health Evaluation, CRRT - continuous renal replacement therapy, ECLS - extracorporeal liver support, GCS - Glasgow Coma Scale, ICU - intensive care unit, INR - prothrombin ratio, IQR - interquartile range, MELD UNOS - Model of End-stage Liver Disease United Network for Organ Sharing, SAPS - Simplified Acute Physiology Score, SD - standard deviation, SOFA - Sequential Organ Failure Assessment.

Table IV. Risk of ICU mortality. AUC characteristics for test variables

\begin{tabular}{|lccccc|}
\hline Test variable & AUC & Standard error & $P$-value & \multicolumn{2}{c|}{$95 \% \mathrm{Cl}$ for AUC } \\
\hline Age & 0.666 & 0.059 & 0.008 & 0.549 & 0.782 \\
\hline SOFA & 0.841 & 0.048 & $<0.001$ & 0.746 & 0.936 \\
\hline APACHE II & 0.832 & 0.044 & $<0.001$ & 0.745 & 0.919 \\
\hline SAPS II & 0.826 & 0.049 & $<0.001$ & 0.730 & 0.921 \\
\hline MELD UNOS & 0.749 & 0.048 & $<0.001$ & 0.654 & 0.843 \\
\hline INR & 0.730 & 0.050 & $<0.001$ & 0.632 & 0.829 \\
\hline Prothrombin time & 0.732 & 0.049 & $<0.001$ & 0.635 & 0.828 \\
\hline Creatinine & 0.743 & 0.053 & $<0.001$ & 0.639 & 0.846 \\
\hline GCS & 0.660 & 0.061 & 0.013 & 0.542 & 0.779 \\
\hline Albumin & 0.728 & 0.055 & $<0.001$ & 0.620 & 0.836 \\
\hline
\end{tabular}

APACHE - Acute Physiology and Chronic Health Evaluation, AUC - area under the curve, Cl - confidence interval, GCS - Glasgow Coma Scale, INR - prothrombin ratio, MELD UNOS - Model of End-stage Liver Disease United Network for Organ Sharing, SAPS - Simplified Acute Physiology Score, SOFA - Sequential Organ Failure Assessment.

Table V. Risk of ICU mortality. Optimum cut-off points and indicators

\begin{tabular}{|c|c|c|c|c|c|c|c|c|}
\hline Test variable & Sensitivity & Specificity & PPV & NPV & OR & $95 \% \mathrm{C}$ & for $O R$ & $P$-value \\
\hline Age $\geq 51.5$ & 0.5075 & 0.7647 & 0.8095 & 0.4407 & 3.3485 & 1.3265 & 8.4523 & 0.0087 \\
\hline SOFA $\geq 10.5$ & 0.9701 & 0.6563 & 0.8553 & 0.9130 & 62.0455 & 12.7167 & 302.7232 & $<0.0001$ \\
\hline APACHE II $\geq 21$ & 0.8507 & 0.7500 & 0.8769 & 0.7059 & 17.1000 & 6.0145 & 48.6174 & $<0.0001$ \\
\hline SAPS II $\geq 39.5$ & 0.9254 & 0.6875 & 0.8611 & 0.8148 & 27.2800 & 8.3944 & 88.6537 & $<0.0001$ \\
\hline $\begin{array}{l}\text { MELD UNOS } \\
\geq 33.5\end{array}$ & 0.5522 & 0.8824 & 0.9024 & 0.5000 & 9.2500 & 2.9317 & 29.1858 & $<0.0001$ \\
\hline INR $\geq 2.41$ & 0.5821 & 0.8529 & 0.8864 & 0.5088 & 8.0786 & 2.7819 & 23.4596 & $<0.0001$ \\
\hline $\begin{array}{l}\text { Prothrombin } \\
\text { time } \geq 31.2\end{array}$ & 0.4925 & 0.9706 & 0.9706 & 0.4925 & 32.0294 & 4.1385 & 247.8866 & $<0.0001$ \\
\hline $\begin{array}{l}\text { Creatinine } \\
\geq 1.83\end{array}$ & 0.6716 & 0.7941 & 0.8654 & 0.5510 & 7.8896 & 2.9752 & 20.9214 & $<0.0001$ \\
\hline $\mathrm{GCS} \leq 14.5$ & 0.8060 & 0.5294 & 0.7714 & 0.5806 & 4.6731 & 1.8893 & 11.5588 & 0.0006 \\
\hline $\begin{array}{l}\text { Albumin } \\
\leq 2.545\end{array}$ & 0.5273 & 0.8750 & 0.8788 & 0.5185 & 7.8077 & 2.4140 & 25.2523 & 0.0002 \\
\hline
\end{tabular}

APACHE - Acute Physiology and Chronic Health Evaluation, Cl - confidence interval, GCS - Glasgow Coma Scale, INR - prothrombin ratio, MELD UNOS - Model of End-stage Liver Disease United Network for Organ Sharing, NPV - negative predictive value, OR - odds ratio, PPV - positive predictive value, SAPS - Simplified Acute Physiology Score, SOFA - Sequential Organ Failure Assessment. 
$(p<0.001 ; \mathrm{OR}=1.188,95 \% \mathrm{Cl}: 1.103-1.280)$, SAPS I

$(p<0.001 ; \mathrm{OR}=1.076,95 \% \mathrm{Cl}: 1.043-1.109), \mathrm{GCS}$ $(p=0.012 ; \mathrm{OR}=0.880,95 \% \mathrm{Cl}: 0.797-0.972)$, MELD UNOS ( $p=0.001$; OR $=1.128,95 \% \mathrm{Cl}: 1.058-$ $1.203)$ scores, INR ( $p=0.001 ; \mathrm{OR}=3.149,95 \% \mathrm{Cl}$ : $1.677-5.915)$, prothrombin time $(p=0.001 ; \mathrm{OR}=$ 1.102 , 95\% Cl: $1.046-1.162)$, creatinine $(p=0.005$; $\mathrm{OR}=1.555,95 \% \mathrm{Cl}: 1.145-2.110)$, albumin ( $p=$ $0.001 ; \mathrm{OR}=0.258,95 \% \mathrm{Cl}: 0.115-0.580), \mathrm{ECLS}$ $(p=0.015 ; \mathrm{OR}=0.346 ; 95 \% \mathrm{Cl}: 0.147-0.816)$, hepatorenal syndrome $(p=0.019 ; \mathrm{OR}=4.717,95 \% \mathrm{Cl}$ : 1.295-17.182), presence of cirrhosis with ascites $(p=0.009 ; \mathrm{OR}=3.129,95 \% \mathrm{Cl}: 1.329-7.366)$ and CRRT ( $p=0.011 ; \mathrm{OR}=3.348,95 \% \mathrm{Cl}: 1.327-8.452)$.

A stepwise (both forward and backward) regression model results in the selection of two variables: SOFA ( $p=0.001 ; \mathrm{OR}=1.451,95 \% \mathrm{Cl}$ : $1.202-1.757)$ and prothrombin time ( $p=0.024$; $\mathrm{OR}=1.085,95 \% \mathrm{Cl}: 1.011-1.165)$ from that set of variables. Each of these variables represents an independent risk factor for ICU mortality.

The model thus obtained is statistically significant: $\chi^{2}=41.17 ; \mathrm{d} f=2 ; p<0.001$. Based on that model, it is possible to correctly classify 20 out of 30 surviving patients (66.7\%), and 50 out of 55 deceased patients (90.9\%)

\section{Risk of 30-day mortality}

Compared to 30-day survivors, the patients who died during the 30-day period were older on ICU admission, had lower GCS scores and lower serum albumin levels, higher SOFA, APACHE II, SAPS $\|$ and MELD UNOS scores, and higher INR, prothrombin time and creatinine levels (Table VI). In the group of patients who died during the 30-day period, ECLS procedures were performed less commonly than CRRT procedures. For the remaining variables, no statistically significant differences were found (Table VI).

Consequently, the following parameters were considered as potential risk factors for 30-day mortality: patient age (years), GCS (score), SOFA (score), APACHE II (score), SAPS II (score), MELD UNOS Modification (score), INR on ICU admission, prothrombin time (s) on ICU admission, creatinine $(\mathrm{mg} / \mathrm{dl})$ on ICU admission, and serum albumin level on ICU admission $(\mathrm{g} / \mathrm{dl})$. For these variables, ROC curves were drawn in order to assess discriminatory power (Table VII).

The highest discriminatory power was shown for the SAPS II score followed by SOFA and APACHE II scores. Age and GCS score were found to have the lowest discriminatory power.

Based on Youden's index, optimum cut-off points were determined. For them, basic performance metrics which evaluate the resulting diagnostic tests suitable for predicting 30-day death were calculated (Table VIII).
The highest values of all four measures (sensitivity, specificity, PPV and NPV) were determined for SAPS II $\geq 39.5$.

With univariate logistic regression, the following risk factors were identified for 30-day mortality in patients with severe liver dysfunction: age ( $p<0.009 ; \mathrm{OR}=1.052,95 \% \mathrm{Cl}: 1.013-1.093)$, SOFA $(p<0.001 ;$ OR $=1.504,95 \% \mathrm{Cl}: 1.265-$ 1.789), APACHE $\|$ ( $p<0.001 ;$ OR $=1.191$, $95 \% \mathrm{Cl}: 1.098-1.291)$, SAPS $\|(p<0.001 ;$ OR $=$ $1.081,95 \% \mathrm{Cl}: 1.045-1.119)$, GCS $(p=0.022$ $\mathrm{OR}=0.878,95 \% \mathrm{Cl}: 0.785-0.981)$, MELD UNOS ( $p=$ $0.001 ; \mathrm{OR}=1.126,95 \% \mathrm{Cl}: 1.050-1.207)$ scores, INR $(p=0.001 ; \mathrm{OR}=3.447,95 \% \mathrm{Cl}: 1.655-7.182)$, prothrombin time $(p=0.001 ; \mathrm{OR}=1.107,95 \% \mathrm{Cl}$ : 1.042-1.176), creatinine ( $p=0.009 ; \mathrm{OR}=1.587$, $95 \% \mathrm{Cl}:$ 1.121-2.248), albumin $(p=0.002$ $\mathrm{OR}=0.237,95 \% \mathrm{Cl}: 0.096-0.585), \mathrm{ECLS}(p=0.027$ $\mathrm{OR}=0.349 ; 95 \% \mathrm{Cl}: 0.137-0.888)$, and CRRT ( $p=$ 0.024; $\mathrm{OR}=3.243,95 \% \mathrm{Cl}: 1.168-9.005)$.

A stepwise (both forward and backward) regression model results in the selection of two variables: SOFA ( $p=0.001 ; \mathrm{OR}=1.440,95 \% \mathrm{Cl}$ : $1.186-1.749)$ and prothrombin time $(p=0.018$ $\mathrm{OR}=1.105,95 \% \mathrm{Cl}: 1.017-1.200)$ from that set of variables. Each of them represents an independent risk factor for 30-day mortality.

The model thus obtained is statistically significant: $\chi^{2}=36.31 \mathrm{~d} f=2 ; p<0.001$. Based on that model, it is possible to correctly classify 15 out of 24 surviving patients (62.5\%), and 55 out of 59 deceased patients (93.2\%).

\section{Discussion}

In our study, short-term mortality was very high $(73.74 \%)$, but in the group of patients receiving standard treatment it was $83.02 \%$, and in the group receiving standard treatment in conjunction with liver dialysis therapy it was $63.04 \%$. The observed high mortality was attributable mainly to the fact that the patients' clinical condition was very severe: the mean values of the SOFA, APACHE II and SAPS II scores determined on admission in the study group were, respectively, 13.68, 25.39 and 54.84 points. Also notable is the high incidence of hepatorenal syndrome (23.76\%), cirrhosis with ascites (59.41\%), alcoholic liver disease (60.20\%) and hepatic encephalopathy (49.00\%) in the study group.

In our study, over $90 \%$ of extracorporeal liver support procedures were liver dialysis treatments based on the SPAD technique. Taking into account results obtained in both subgroups (Table II), it needs to be concluded that SPAD is an effective method of eliminating bilirubin and ammonia.

In the present study, the SOFA, APACHE II, and SAPS II scores were shown to be better predictors of death than the MELD UNOS Modification 
Table VI. 30-day mortality. Descriptive characteristics of survivors and deceased patients

\begin{tabular}{|c|c|c|c|c|}
\hline Variable & & $\begin{array}{l}\text { Patients who survived } \\
30 \text { days }(N=26)\end{array}$ & $\begin{array}{l}\text { Patients who died during } \\
30 \text { days }(N=67)\end{array}$ & $P$-value \\
\hline Age [years] & $\begin{array}{l}\text { Mean } \pm \text { SD } \\
\text { Me (IQR) } \\
\text { Min.-max. }\end{array}$ & $\begin{array}{c}42.04 \pm 13.56 \\
40.5(31-51) \\
24-78\end{array}$ & $\begin{array}{c}50.14 \pm 12.59 \\
51(43-58) \\
25.83\end{array}$ & 0.0069 \\
\hline $\begin{array}{l}\text { Hepatic } \\
\text { encephalopathy }\end{array}$ & $N(\%)$ & $9(34.62)$ & $40(55.56)$ & 0.0655 \\
\hline $\begin{array}{l}\text { Cirrhosis with } \\
\text { ascites }\end{array}$ & $N(\%)$ & $12(46.15)$ & $48(65.75)$ & 0.0790 \\
\hline $\begin{array}{l}\text { Hepatorenal } \\
\text { syndrome }\end{array}$ & $N(\%)$ & $3(11.54)$ & $21(28.77)$ & 0.1352 \\
\hline $\begin{array}{l}\text { Alcoholic liver } \\
\text { disease }\end{array}$ & $N(\%)$ & $17(68.00)$ & $42(59.15)$ & 0.4307 \\
\hline GCS score & $\begin{array}{l}\text { Mean } \pm \text { SD } \\
\text { Me (IQR) } \\
\text { Min.-max. }\end{array}$ & $\begin{array}{c}12.27 \pm 4.08 \\
15(9-15) \\
3-15\end{array}$ & $\begin{array}{c}9.71 \pm 4.79 \\
11(5-14) \\
3-15\end{array}$ & 0.0041 \\
\hline SOFA score & $\begin{array}{l}\text { Mean } \pm \text { SD } \\
\text { Me (IQR) } \\
\text { Min.-max. }\end{array}$ & $\begin{array}{c}9.76 \pm 4.01 \\
8(7-12) \\
5-17\end{array}$ & $\begin{array}{c}15.26 \pm 3.41 \\
15(13-17.5) \\
7-22\end{array}$ & $<0.0001$ \\
\hline APACHE II score & $\begin{array}{l}\text { Mean } \pm \text { SD } \\
\text { Me (IQR) } \\
\text { Min.-max. }\end{array}$ & $\begin{array}{c}17.56 \pm 7.61 \\
17(13-20) \\
6-36\end{array}$ & $\begin{array}{c}28.57 \pm 8.84 \\
28(22.5-34) \\
12-55\end{array}$ & $<0.0001$ \\
\hline SAPS II score & $\begin{array}{l}\text { Mean } \pm \text { SD } \\
\text { Me (IQR) } \\
\text { Min.-max. }\end{array}$ & $\begin{array}{c}36.00 \pm 18.83 \\
29(24-39) \\
15-81\end{array}$ & $\begin{array}{c}62.08 \pm 18.86 \\
63(48-73) \\
22-103\end{array}$ & $<0.0001$ \\
\hline $\begin{array}{l}\text { MELD UNOS } \\
\text { Modification } \\
\text { score }\end{array}$ & $\begin{array}{l}\text { Mean } \pm \text { SD } \\
\text { Me (IQR) } \\
\text { Min.-max. }\end{array}$ & $\begin{array}{c}27.00 \pm 5.49 \\
26.5(23-30) \\
18-40 \\
\end{array}$ & $\begin{array}{c}33.56 \pm 8.20 \\
34(27-40) \\
18-48 \\
\end{array}$ & 0.0005 \\
\hline $\begin{array}{l}\text { Length of } \\
\text { hospital stay } \\
\text { [days] }\end{array}$ & $\begin{array}{l}\text { Mean } \pm \text { SD } \\
\text { Me (IQR) } \\
\text { Min.-max. }\end{array}$ & $\begin{array}{c}28.12 \pm 18.25 \\
27(13-46) \\
5-60\end{array}$ & $\begin{array}{c}18.67 \pm 15.29 \\
15(7-28) \\
1-63\end{array}$ & 0.0193 \\
\hline $\begin{array}{l}\text { Length of ICU } \\
\text { stay [days] }\end{array}$ & $\begin{array}{l}\text { Mean } \pm \text { SD } \\
\text { Me (IQR) } \\
\text { Min.-max. }\end{array}$ & $\begin{array}{c}12.42 \pm 12.58 \\
7(5-14) \\
3-48\end{array}$ & $\begin{array}{c}10.37 \pm 10.00 \\
8(3-13) \\
1-51\end{array}$ & 0.6128 \\
\hline $\begin{array}{l}\text { Total bilirubin } \\
{[\mathrm{mg} / \mathrm{dl}]}\end{array}$ & $\begin{array}{l}\text { Mean } \pm \text { SD } \\
\text { Me (IQR) } \\
\text { Min.-max. }\end{array}$ & $\begin{array}{c}21.66 \pm 13.79 \\
20.76(9.02-32.63) \\
0.66-45.54\end{array}$ & $\begin{array}{c}17.52 \pm 11.97 \\
17.51(6.83-26.64) \\
0.69-43.22\end{array}$ & 0.1895 \\
\hline $\begin{array}{l}\text { Direct bilirubin } \\
{[\mathrm{mg} / \mathrm{dl}]}\end{array}$ & $\begin{array}{l}\text { Mean } \pm \text { SD } \\
\text { Me (IQR) } \\
\text { Min.-max. }\end{array}$ & $\begin{array}{c}24.23 \pm 10.37 \\
22.56(15.51-32.28) \\
2.08-43.21\end{array}$ & $\begin{array}{c}20.06 \pm 8.98 \\
21.16(13.24-27.4) \\
0.71-42.85\end{array}$ & 0.1271 \\
\hline $\begin{array}{l}\text { Bile acids } \\
{[\mu \mathrm{mol} / \mathrm{l}]}\end{array}$ & $\begin{array}{l}\text { Mean } \pm \text { SD } \\
\text { Me (IQR) } \\
\text { Min.-max. }\end{array}$ & $\begin{array}{c}119.68 \pm 107.24 \\
95.05(77.5-127.25) \\
28.7-549.3\end{array}$ & $\begin{array}{c}101.06 \pm 75.88 \\
80.4(51.8-138.7) \\
28.4-415.8 \\
\end{array}$ & 0.3238 \\
\hline $\begin{array}{l}\text { Ammonia } \\
{[\mu \mathrm{g} / \mathrm{dl}]}\end{array}$ & $\begin{array}{l}\text { Mean } \pm \text { SD } \\
\text { Me }(I Q R) \\
\text { Min.-max. }\end{array}$ & $\begin{array}{c}93.76 \pm 39.10 \\
92(67-119) \\
23-165\end{array}$ & $\begin{array}{c}174.17 \pm 227.60 \\
108(73-165) \\
13-1535\end{array}$ & 0.1334 \\
\hline INR & $\begin{array}{l}\text { Mean } \pm \text { SD } \\
\text { Me (IQR) } \\
\text { Min.-max. }\end{array}$ & $\begin{array}{c}1.89 \pm 0.48 \\
1.915(1.5-2.31) \\
1.05-2.7\end{array}$ & $\begin{array}{c}2.64 \pm 0.97 \\
2.52(1.8-3.33) \\
1.24-5.46\end{array}$ & 0.0005 \\
\hline $\begin{array}{l}\text { Prothrombin } \\
\text { time [s] }\end{array}$ & $\begin{array}{l}\text { Mean } \pm \text { SD } \\
\text { Me (IQR) } \\
\text { Min.-max. }\end{array}$ & $\begin{array}{c}22.10 \pm 5.39 \\
22.35(17.2-26.5) \\
12.1-31.1 \\
\end{array}$ & $\begin{array}{c}33.55 \pm 22.05 \\
30.04(20.9-42.1) \\
14.4-189.7 \\
\end{array}$ & 0.0005 \\
\hline $\begin{array}{l}\text { Creatinine } \\
{[\mathrm{mg} / \mathrm{dl}]}\end{array}$ & $\begin{array}{l}\text { Mean } \pm \text { SD } \\
\text { Me (IQR) } \\
\text { Min.-max. }\end{array}$ & $\begin{array}{c}1.59 \pm 1.45 \\
1(0.75-1.71) \\
0.4-6.5\end{array}$ & $\begin{array}{c}2.97 \pm 2.42 \\
2.43(1.22-4.12) \\
0.1-13.52 \\
\end{array}$ & 0.0014 \\
\hline Albumin [g/dl] & $\begin{array}{l}\text { Mean } \pm \text { SD } \\
\text { Me (IQR) } \\
\text { Min.-max. }\end{array}$ & $\begin{array}{c}3.12 \pm 0.50 \\
3.11(2.85-3.49) \\
2.11-4.15\end{array}$ & $\begin{array}{c}2.60 \pm 0.67 \\
2.565(2.105-3.10) \\
0.9-4.16\end{array}$ & 0.0007 \\
\hline
\end{tabular}


Table VI. Cont.

\begin{tabular}{|lccc|}
\hline Variable & $\begin{array}{c}\text { Patients who survived } \\
\text { 30 days }(N=26)\end{array}$ & $\begin{array}{c}\text { Patients who died during } \\
\text { 30 days }(N=67)\end{array}$ & P-value \\
\hline $\begin{array}{l}\text { ECLS during ICU } \\
\text { stay }\end{array}$ & $N(\%)$ & $29(39.73)$ & 0.0238 \\
\hline $\begin{array}{l}\text { CRRT during ICU } \\
\text { stay }\end{array}$ & $N(\%)$ & $36(49.38)$ & 0.0171 \\
\hline $\begin{array}{l}\text { APACHE - Acute Physiology and Chronic Health Evaluation, CRRT - continuous renal replacement therapy, ECLS - extracorporeal liver } \\
\text { support, GCS - Glasgow Coma Scale, ICU - intensive care unit, INR - prothrombin ratio, IQR - interquartile range, MELD UNOS - Model } \\
\text { of End-stage Liver Disease United Network for Organ Sharing, SAPS - Simplified Acute Physiology Score, SD - standard deviation, SOFA }\end{array}$ \\
- - Sequential Organ Failure Assessment.
\end{tabular}

Table VII. Risk of 30-day mortality. AUC characteristics for test variables

\begin{tabular}{|lccccc|}
\hline Test variable & AUC & Standard error & $P$-value & \multicolumn{2}{c|}{$95 \%$ Cl for AUC } \\
\hline Age & 0.670 & 0.064 & 0.011 & 0.545 & 0.796 \\
\hline GCS & 0.687 & 0.061 & 0.005 & 0.567 & 0.807 \\
\hline SOFA & 0.834 & 0.053 & $<0.001$ & 0.731 & 0.937 \\
\hline APACHE II & 0.828 & 0.049 & $<0.001$ & 0.732 & 0.924 \\
\hline SAPS II & 0.838 & 0.054 & $<0.001$ & 0.733 & 0.944 \\
\hline MELD UNOS & 0.731 & 0.052 & 0.001 & 0.630 & 0.833 \\
\hline INR & 0.743 & 0.050 & $<0.001$ & 0.646 & 0.840 \\
\hline Prothrombin time & 0.743 & 0.049 & $<0.001$ & 0.647 & 0.838 \\
\hline Creatinine & 0.702 & 0.060 & 0.003 & 0.585 & 0.820 \\
\hline Albumin & 0.751 & 0.054 & $<0.001$ & 0.645 & 0.856 \\
\hline
\end{tabular}

APACHE - Acute Physiology and Chronic Health Evaluation, AUC - area under the curve, Cl - confidence interval, GCS - Glasgow Coma Scale, INR - prothrombin ratio, MELD UNOS - Model of End-stage Liver Disease United Network for Organ Sharing, SAPS - Simplified Acute Physiology Score, SOFA - Sequential Organ Failure Assessment.

Table VIII. Risk of 30-day mortality. Optimum cut-off points and indicators

\begin{tabular}{|lcccccccc|}
\hline Variable & Sensitivity & Specificity & PPV & NPV & OR & \multicolumn{2}{c|}{$95 \%$ Cl for OR } & $P$-value \\
\hline Age $\geq 46.5$ & 0.6301 & 0.7308 & 0.8679 & 0.4130 & 4.6243 & 1.7212 & 12.4243 & 0.0015 \\
\hline SOFA $\geq 12.5$ & 0.8194 & 0.7600 & 0.9077 & 0.5938 & 14.3718 & 4.7991 & 43.0394 & $<0.0001$ \\
\hline APACHE II $\geq 21$ & 0.8194 & 0.7600 & 0.9077 & 0.5938 & 14.3718 & 4.7991 & 43.0394 & $<0.0001$ \\
\hline SAPS II $\geq 39.5$ & 0.9028 & 0.7600 & 0.9155 & 0.7308 & 29.4048 & 8.8185 & 98.0487 & $<0.0001$ \\
\hline $\begin{array}{l}\text { MELD UNOS } \\
\geq 31.5\end{array}$ & 0.6164 & 0.8077 & 0.9000 & 0.4286 & 6.7500 & 2.2844 & 19.9449 & 0.0002 \\
\hline INR $\geq 2.71$ & 0.4658 & 1.0000 & 1.0000 & 0.4000 & $0 R$ could not be determined & $<0.0001$ \\
\hline $\begin{array}{l}\text { Prothrombin } \\
\text { time } \geq 29.75\end{array}$ & 0.5205 & 0.9615 & 0.9744 & 0.4167 & 27.1429 & 3.4912 & 211.0292 & $<0.0001$ \\
\hline $\begin{array}{l}\text { Creatinine } \\
\geq 1.81\end{array}$ & 0.6575 & 0.8077 & 0.9057 & 0.4565 & 8.0640 & 2.7152 & 23.9499 & $<0.0001$ \\
\hline GCS $\leq 14.5$ & 0.7945 & 0.5385 & 0.8286 & 0.4828 & 4.5111 & 1.7315 & 11.7527 & 0.0014 \\
\hline $\begin{array}{l}\text { Albumin } \\
\leq 2.935\end{array}$ & 0.6667 & 0.7200 & 0.8511 & 0.4737 & 5.1429 & 1.8455 & 14.3317 & 0.0011 \\
\hline
\end{tabular}

APACHE - Acute Physiology and Chronic Health Evaluation, Cl - confidence interval, GCS - Glasgow Coma Scale, INR - prothrombin ratio, MELD UNOS - Model of End-stage Liver Disease United Network for Organ Sharing, NPV - negative predictive value, OR - odds ratio, PPV - positive predictive value, SAPS - Simplified Acute Physiology Score, SOFA - Sequential Organ Failure Assessment. 
score, which is dedicated to the assessment of patients with liver disease. The findings are consistent with our previous studies and reports by other authors [21].

Orthotopic liver transplant is the only method which contributes to prolonging the survival of patients with liver dysfunction. The overall post-transplant survival rate is $84 \%$, and in patients with ALF it reaches even 92\% [17]. Liver transplantation, however, is not a widely accessible therapeutic method. Its limited accessibility results on the one hand from a low availability of organs for transplantation, and on the other hand from indications and contraindications involved in the application of the procedure in a particular patient.

If the transplant option is unavailable, there are no clinically effective treatment methods that can be used in patients with severe liver dysfunction (MELD UNOS score $\geq 18$ ), which results in poor predicted prognosis [12].

Some hope, at least theoretically, can be placed on extracorporeal liver support techniques which help to win additional time, so that patients are able to regenerate damaged liver in cases where such regeneration is potentially possible.

There are only scarce data about the application of FPSA in patients with severe liver dysfunction. The first uncontrolled study conducted in 11 patients with acute-on-chronic liver failure (ACLF) and coexisting renal insufficiency showed FPSA to significantly reduce the level of conjugated bilirubin in the serum, and the levels of bile acids, ammonia, cholinesterase, creatinine and urea [23]. There were no significant changes in the level of haemoglobin and blood platelet count, while the leukocyte count was found to have increased [23]. The findings were corroborated in another uncontrolled study [24].

The study by Kribben et al. failed to demonstrate any differences in short-term and long-term mortality [12]. The probability of 28-day survival in the FPSA group was 66\%, and 90-day survival was $47 \%$. In contrast, the probability of 28 -day survival in the SMT group was $63 \%$, and 90-day survival was $38 \%$. The difference was noticeable, but statistically insignificant. It was significant only in the subgroup of patients whose MELD score was above 30. A multivariate analysis also demonstrated better survival rates in FPSA-treated patients with hepatorenal syndrome type 1 . The same finding was obtained for patients with alcoholic liver cirrhosis. The treatment triggered a significant decrease in bilirubin concentration. No statistical differences were noted for the remaining parameters. The authors of the study claimed that the result may have been influenced by the fact that the study group comprised patients with end-stage liver cirrhosis which was irreversible. The authors had no specific tool for differentiating between patients with end-stage liver failure and ACLF which is potentially reversible even with standard treatment methods.

A metaanalysis of four randomized and two selected non-randomized studies involving the administration of MARS to ACLF patients failed to demonstrate any effect on mortality $[13,14$, 25-29]. An in-depth analysis of non-randomized studies, however, corroborated a significant decrease in mortality in the MARS group compared to the group of patients who received standard treatment [25]. A recently published observational study highlights a higher incidence of survival in ALF patients without a transplant compared to expected values [30]. In ALF syndrome induced by drugs (paracetamol) or toxins (mushroom poisoning), MARS yielded a beneficial effect observed in a larger population of patients (a non-randomized study) [31, 32].

A metaanalysis comprising 10 randomized clinical studies, of which four assessed the usefulness of MARS in ALF (93 patients) and six in ACLF (453 patients), compared the effects of MARS therapy with standard treatment. MARS was shown to prolong the survival of ALF patients, but no evidence that it improved survival in patients with ACLF was found [33].

The MARS turned out to be an effective method in decreasing portal hypertension, increasing circulatory efficiency and reducing the degree of hepatic encephalopathy. By eliminating endogenous substances which accumulate during insufficiency of the liver, and hence the kidneys, and impair the metabolism and haemodynamics, it was found to improve circulatory efficiency in patients with advanced liver cirrhosis. The development of a systemic inflammatory response was shown to have a potential to induce a progression from stable liver cirrhosis and lead to organ damage. Inflammation and oxidative stress may result in increased production of NO, which leads to circulatory and renal insufficiency and, as a consequence, impairs liver function [15]. Several studies, including small randomized studies, have shown albumin dialysis to be an effective method of prolonging survival, decreasing the concentration of bilirubin and degree of hepatic encephalopathy, and improving circulatory and renal function in patients with ACLF [15].

A study comparing FPSA with MARS demonstrated FPSA to have higher efficacy in eliminating bilirubin and urea, and comparable efficacy in eliminating bile acids. However, only MARS has been shown to significantly attenuate hyperdynamic circulation in patients with $\operatorname{ACLF}[7,34,35]$.

In case reports and small-scale clinical trials, researchers have noted that SPAD albumin dialysis brings down the concentration of bilirubin and im- 
proves the clinical condition of patients, especially in ALF induced by toxins (e.g. paracetamol) and in Wilson's disease [36-39].

The SPAD-based liver dialysis should be performed with a dialysate flow rate of at least 1,000 $\mathrm{ml} / \mathrm{h}$ [40]. The higher the dialysate flow rate, the greater the efficacy of the dialysis procedure manifested by a decrease in the plasma concentrations of bilirubin, bile acids and ammonia [40]. Furthermore, provided that the dialysate flow is properly adjusted depending on the patient, the risk of metabolic and electrolyte disorders reported by Sponholz et al. during citrate anticoagulation is very low [40, 41].

$A$ vast majority of patients with severe liver dysfunction accompanied by the failure of several other organs have a poor prognosis regardless of whether extracorporeal liver support (Prometheus, MARS or SPAD) is administered or not. The 30-day mortality rate in this patient group often exceeds $70 \%[1,42]$.

In spite of proven biochemical efficacy of non-biological extracorporeal liver support techniques, the data on clinical endpoints are very scanty. In their metaanalysis, Stutchfield et al. as sessed the effect of extracorporeal liver support on the survival of patients with ALF and ACLF [43]. Extracorporeal liver support prolongs patient survival in ALF (RR 0.70, 95\% Cl: 0.49-1.00). Number needed to treat (NNT), i.e. the number of patients who must undergo a particular intervention for a defined time to prevent one unfavourable endpoint, was eight. No significant differences in mortality were found between extracorporeal liver support and standard medical therapy in ACLF $(\mathrm{RR}=0.87,95 \% \mathrm{Cl}: 0.64-1.18)$ [43].

The ALF patients seem to be the most appropriate patient group for treatment based on non-biological extracorporeal liver support techniques, because in these patients there is a potential for complete liver regeneration (i.e. restoration of the initial condition). Nevertheless, extracorporeal liver support techniques are most commonly administered to patients with chronic liver damage, often at the cirrhosis stage. In this case, severe liver dysfunction (MELD UNOS $\geq 18$ ) is often associated with the failure of other organs and high mortality rates. In end-stage liver cirrhosis, non-biological extracorporeal liver support should be restricted to patients who are on the transplant waiting list.

Depending on the degree of liver insufficiency, short-term (28-day) mortality among ACLF patients ranges from $22 \%$ to $73 \%$, and in patients with chronic insufficiency without exacerbations it is $4.9 \%[44,45]$.

The main limitation of the present study is its retrospective nature.

The results obtained in the study were affected by the fact that even though there was no statistical difference between the two study subgroups
(SMT and SMT + ECLS) in terms of the basic criterion, i.e. the MELD UNOS score, the subgroups differed significantly in some important parameters. SMT was a subgroup comprising older patients who had lower GCS scores and higher SOFA, APACHE II and SAPS II scores, and higher INR and creatinine levels than those in the SMT + ECLS subgroup. On the other hand, SMT + ECLS subgroup patients were more commonly diagnosed with hepatorenal syndrome, and had higher total and direct bilirubin levels on ICU admission. In view of the above, the lower mortality level observed in the SMT + ECLS subgroup in comparison to SMT may not be a result of treatment but rather a consequence of differences in the clinical condition of the patients in both subgroups reflected by their SOFA, APACHE II and SAPS II scores. The statistical analysis performed for the study showed that the SOFA score was an independent risk factor for ICU mortality and 30-day mortality. A one-point increase in the SOFA score increases the risk of ICU mortality by $45.1 \%$, and the risk of 30 -day mortality by $44 \%$. These findings are consistent with the outcomes reported by Jalan et al., who found that the SOFA and APACHE II scores had the highest prognostic predictive value for mortality [44].

However, taking into account the logistic regression model estimates calculated individually for each variable, it cannot be ruled out that the decrease in mortality was a consequence of performing extracorporeal liver support procedures in the SMT + ECLS subgroup. A univariate logistic regression model showed that ECLS procedures reduced the risk of ICU mortality ( $p=0.015$; $\mathrm{OR}=0.346,95 \% \mathrm{Cl}: 0.147-0.816)$ and 30-day mortality $(p=0.027$; OR $=0.349,95 \% \mathrm{Cl}: 0.137-$ 0.888). The effect, however, was not confirmed in a multivariate model. Further studies, conducted in a larger population of patients, are needed to assess the benefit of ECLS procedures in patients with severe liver dysfunction.

In conclusion, extracorporeal liver support techniques (SPAD, FPSA) effectively eliminate bilirubin and ammonia in patients with severe liver dysfunction (MELD UNOS $\geq 18$ ). Further studies are needed in order to assess the contribution of non-biological extracorporeal liver support procedures to a decrease in mortality rates in the group of patients with severe liver dysfunction. Independent risk factors for ICU mortality and 30day mortality were shown to be the SOFA score and prothrombin time. The SOFA, APACHE II, and SAPS II scores were shown to be better predictors of death than the MELD UNOS Modification score, which is dedicated to the assessment of patients with liver disease.

\section{Conflict of interest}

The authors declare no conflict of interest. 


\section{References}

1. EASL clinical practical guidelines: management of alcoholic liver disease. J Hepatol 2012; 57: 399-420.

2. Mathurin P, Lucey MR. Management of alcoholic hepatitis. J Hepatol 2012; 56 Suppl. 1: 39-45.

3. Oshea RS, Dasarathy S, McCullough AJ. Alcoholic liver disease. Am J Gastroenterol 2010; 105: 14-32.

4. Jalan R, Sen S, Steiner C, Kapoor D, Alisa A, Williams R. Extracorporeal liver support with molecular adsorbents recirculating system in patients with severe acute alcoholic hepatitis. J Hepatol 2003; 38: 24-31.

5. Sen S, Mookerjee RP, Cheshire LM, Davies NA, Williams R, Jalan R. Albumin dialysis reduces portal pressure acutely in patients with severe alcoholic hepatitis. J Hepatol 2005; 43: 142-8.

6. Catalina MV, Barrio J, Anaya F, et al. Hepatic and systemic haemodynamic changes after MARS in patients with acute on chronic liver failure. Liver Int 2003; 23 Suppl. 3: 39-43.

7. Laleman W, Wilmer A, Evenepoel P, et al. Effect of the molecular adsorbent recirculating system and Prometheus devices on systemic haemodynamics and vasoactive agents in patients with acute-on-chronic alcoholic liver failure. Crit Care 2006; 10: R108.

8. Parés A, Cisneros L, Salmerón JM, et al. The molecular adsorbent recirculating system (MARS) improves systemic hemodynamics and hepatic encephalopathy in patients with severe alcoholic hepatitis. J Hepatol 2002; 36 Suppl. 1: 150.

9. Parés A, Deulofeu R, Cisneros L, et al. Albumin dialysis improves hepatic encephalopathy and decreases circulating phenolic aromatic amino acids in patients with alcoholic hepatitis and severe liver failure. Crit Care 2009; 13: R8.

10. Hassanein T, Tamsen MR, Petcharaporn L, Hassanemin R, Monson P, Baralak F. Extracorporeal albumin dialysis improves survival in patients with severe alcoholic hepatitis. Hepatology 2012; 56: 974A.

11. Bañares R, Nevens F, Larsen FS, et al. Extracorporeal albumin dialysis with the molecular adsorbent recirculating system in acute-on-chronic liver failure: the RELIEF trial. Hepatology 2013; 57: 1153-62.

12. Kribben A, Gerken G, Haag S, et al. Effects of fractionated plasma separation and adsorption on survival in patients with acute-on-chronic liver failure. Gastroenterology 2012; 142: 782-9.e3.

13. Heemann U, Treichel U, Loock J, et al. Albumin dialysis in cirrhosis with superimposed acute liver injury: a prospective, controlled study. Hepatology 2002; 36: 949-58.

14. El Banayosy A, Kizner L, Schueler V, Bergmeier S, Cobaugh $D$, Koerfer R. First use of the molecular adsorbent recirculating system technique on patients with hypoxic liver failure after cardiogenic shock. ASAIO I 2004; 50: 332-7.

15. Sen S, Davies NA, Mookerjee RP, et al. Pathophysiological effects of albumin dialysis in acute-on-chronic liver failure: a randomized controlled study. Liver Transplant 2004; 10: 1109-19.

16. Ellis AJ, Hughes RD, Wendon JA, et al. Pilot-controlled trial of the extracorporeal liver assist device in acute liver failure. Hepatology 1996; 24: 1446-51.

17. Demetriou AA, Brown Jr RS, Busuttil RW, et al. Prospective, randomized, multicenter, controlled trial of a bioartificial liver in treating acute liver failure. Ann Surg 2004; 239: 660-70.

18. Saliba F, Camus C, Durand F, et al. Albumin dialysis with a noncell artificial liver support device in patients with acute liver failure: a randomized, controlled trial. Ann Intern Med 2013; 159: 522-31.

19. Hassanein TI, Tofteng F, Brown Jr RS, et al. Randomized controlled study of extracorporeal albumin dialysis for hepatic encephalopathy in advanced cirrhosis. Hepatology 2007; 46: 1853-62.

20. Dethloff T, Tofteng F, Frederiksen HJ, Hojskov M, Hansen BA, Larsen FS. Effect of Prometheus liver assist system on systemic hemodynamics in patients with cirrhosis: a randomized controlled trial study. World J Gastroenterol 2008; 14: 2065-71.

21. Piechota M, Piechota A. An evaluation of the usefulness of extracorporeal liver support techniques in patients hospitalized in the ICU for severe liver dysfunction secondary to alcoholic liver disease. Hepat Mon 2016; 16: e34127.

22. Falkenhagen D, Strobl W, Vogt G, et al. Fractionated plasma separation and adsorption system: a novel system for blood purification to remove albumin bound substances. Artif Organs 1999; 23: 81-6.

23. Rifai K, Ernst T, Kretschmer U, et al. Prometheus ${ }^{\oplus}-$ a new extracorporeal system for the treatment of liver failure. J Hepatol 2003; 39: 984-90.

24. Evenepoel P, Laleman W, Wilmer A, et al. Detoxifying capacity and kinetics of Prometheus ${ }^{\oplus}-$ a new extracorporeal system for the treatment of liver failure. Blood Purification 2005; 23: 349-58.

25. Khuroo MS, Khuroo MS, Farahat KLC. Molecular adsorbent recirculating system for acute and acute-on-chronic liver failure: a meta-analysis. Liver Transplant 2004; 10: 1099-106.

26. Mitzner SR, Klammt S, Peszynski P, et al. Improvement of multiple organ functions in hepatorenal syndrome during albumin dialysis with the Molecular Adsorbent Recirculating System. Therap Apheresis 2001; 5: 417-22.

27. Schmidt LE, Wang LP, Hansen BA, Larsen FS. Systemic hemodynamic effects of treatment with the molecular adsorbents recirculating system in patients with hyperacute liver failure: a prospective controlled trial. Liver Transplant 2003; 9: 290-297.

28. Chen S, Zhang L, Shi Y, Yang X, Wang M. Molecular adsorbent recirculating system: clinical experience in patients with liver failure based on hepatitis B in China. Liver 2002; 22 Suppl. 2: 48-51.

29. Hessel FP, Mitzner SR, Rief J, Guellstorff B, Steiner S, Wasem J. Economic evaluation and 1-year survival analysis of MARS in patients with alcoholic liver disease. Liver International 2003; 23 Suppl. 3: 66-72.

30. Camus C, Lavoué S, Gacouin A, et al. Molecular adsorbent recirculating system dialysis in patients with acute liver failure who are assessed for liver transplantation. Intensive Care Med 2006; 32: 1817-25.

31. Wittebole X, Hantson P. Use of the molecular adsorbent recirculating system $\left(\right.$ MARS $\left.^{\mathrm{TM}}\right)$ for the management of acute poisoning with or without liver failure. Clin Toxicol 2011; 49: 782-93.

32. Zhou XM, Miao JY, Yang Y, et al. Clinical experience with molecular adsorbent recirculating system (MARS) in patients with drug-induced liver failure. Artif Organs 2004; 28: 483-6.

33. He GL, Feng L, Duan CY, et al. Meta-analysis of survival with the molecular adsorbent recirculating system for liver failure. Int J Clin Exp Med 2015; 8: 17046-54.

34. Krisper P, Haditsch B, Stauber R, et al. In vivo quantification of liver dialysis: comparison of albumin dialysis and fractionated plasma separation. J Hepatol 2005; 43: 451-7. 
35. Stadlbauer V, Krisper P, Beuers U, et al. Removal of bile acids by two different extracorporeal liver support systems in acute-on-chronic liver failure. ASAIO J 2007; 53: 187-93.

36. Rademacher S, Oppert M, Jörres A. Artificial extracorporeal liver support therapy in patients with severe liver failure. Exp Rev Gastroenterol Hepatol 2011; 5: 591-9.

37. Karvellas CJ, Bagshaw SM, McDermid RC, Stollery DE, Gibney RT. Acetaminophen-induced acute liver failure treated with single-pass albumin dialysis: report of a case. Intern J Artif Organs 2008; 31: 450-5.

38. Collins KL, Roberts EA, Adeli K, Bohn D, Harvey EA. Single pass albumin dialysis (SPAD) in fulminant Wilsonian liver failure: a case report. Pediatr Nephrol 2008; 23 : 1013-6.

39. Piechota M. Hepatic encephalopathy in the course of alcoholic liver disease - treatment options in the intensive care unit. Anaesthesiol Intensive Ther 2014; 46: 34-6.

40. Piechota M, Piechota A. An evaluation of the usefulness of single pass albumin dialysis: key role of dialysate flow rate. Crit Care 2016; 20: 156.

41. Sponholz C, Matthes K, Rupp D, Backaus W, Klammt S, Karailieva D. Molecular adsorbent recirculating system and single-pass albumin dialysis in liver failure - a prospective, randomised crossover study. Crit Care 2016; 20: 2.

42. O'Shea RS, Dasarathy S, McCullough AJ, et al. Alcoholic liver disease. Hepatology 2010; 51: 307-28.

43. Stutchfield BM, Simpson K, Wigmore SJ. Systematic review and meta-analysis of survival following extracorporeal liver support. Br J Surg 2011; 98: 623-31.

44. Jalan R, Gines P, Olson JC, et al. Acute-on chronic liver failure. J Hepatol 2012; 57: 1336-48.

45. Arroyo V, Moreau R, Jalan R, Ginès P. Acute-on-chronic liver failure: a new syndrome that will re-classify cirrhosis. J Hepatol 2015; 62 Suppl. 1: S131-43. 